Comparison of non-Markovianity criteria in a qubit system under random external fields

This content has been downloaded from IOPscience. Please scroll down to see the full text. 2013 Phys. Scr. 2013014047

(http://iopscience.iop.org/1402-4896/2013/T153/014047)

View the table of contents for this issue, or go to the journal homepage for more

Download details:

IP Address: 81.57.242.12

This content was downloaded on 04/03/2014 at 13:38

Please note that terms and conditions apply. 


\title{
Comparison of non-Markovianity criteria in a qubit system under random external fields
}

\author{
Maria Mannone, Rosario Lo Franco and Giuseppe Compagno \\ Dipartimento di Fisica, Università di Palermo, via Archirafi 36, I-90123 Palermo, Italy \\ E-mail: rosario.lofranco@unipa.it
}

Received 3 August 2012

Accepted for publication 9 September 2012

Published 28 March 2013

Online at stacks.iop.org/PhysScr/T153/014047

\begin{abstract}
We give the map representing the evolution of a qubit under the action of non-dissipative random external fields. From this map, we construct the corresponding master equation that in turn allows us to phenomenologically introduce population damping of the qubit system. We then compare, in this system, the time regions where non-Markovianity is present on the basis of different criteria for both the non-dissipative and the dissipative case. We show that the adopted criteria agree both in the non-dissipative case and in the presence of population damping.
\end{abstract}

(Some figures may appear in colour only in the online journal)

\section{Introduction}

In quantum systems, the dynamics of decoherence, and that of quantum correlations, is qualitatively different if the environment is Markovian (without memory) or non-Markovian (with memory) [1-3]. For example, for composite quantum systems independent non-Markovian environments, entanglement may present revivals [4-6] or trapping [7, 8], defending it against sudden death [9]. Non-Markovian systems are utilized in several physical contexts such as quantum optics [1], solid-state physics [10], quantum chemistry [11] and quantum information processing [12]. It is therefore essential to establish criteria for identifying and quantifying the non-Markovian behavior in an open quantum system. Among the criteria, the one introduced by Breuer-Laine-Piilo (BLP) is based on the concept of temporary flow of information from the environment back into the system and quantifies non-Markovianity as an increase in the distinguishability of two evolving quantum states [13]. A second one, due to Rivas-Huelga-Plenio (RHP), instead measures the deviation of the dynamical map from divisibility [14]. A third one has also been proposed by Andersson-Cresser-Hall (ACH) that uses the negative decoherence rates appearing in the master equation as a primary measure to completely characterize non-Markovianity [15]. An all-optical experiment has recently been developed to control transitions from Markovian to non-Markovian dynamics [16].

A natural question is then whether the different criteria agree in identifying non-Markovian behaviors in the system dynamics. It has been shown that, for a qubit coupled to environments via the Jaynes-Cummings or dephasing models, the BLP and RHP criteria have exactly the same non-Markovian time-evolution intervals and are therefore equivalent [17]. In an analysis performed for a driven qubit in a structured environment it has been suggested that the two measures may disagree [18] and successively it has been shown for both a classical and a quantum toy model [3]. Comparisons among the three criteria, including the $\mathrm{ACH}$ one, showing possible non-equivalence in realistic systems are instead still missing.

In this paper we address this issue. In particular, our aim is to verify, for a realistic physical system made of a qubit subject to random external fields both with and without dissipation, whether the BLP, RHP and $\mathrm{ACH}$ criteria give concordant results in individuating the non-Markovian time regions in the system dynamics.

\section{The model}

We consider a realistic system made of a qubit subject to random external fields both in a non-dissipative and in a dissipative case. In the following, we describe the two cases. 


\subsection{Non-dissipative random external fields}

Our system is a qubit interacting with an environment composed of a classical field mode with fixed amplitude but with random phase equal either to zero or to $\pi$ with probability $p=1 / 2$. This model has been introduced to study the possibility of revivals of quantum correlations in the absence of back-action [19] and describes a special case of a qubit subject to a phase noisy laser [20,21]. The dynamical map is of the random external fields type $[22,23]$ and, in the qubit basis $\{|1\rangle,|2\rangle\}$, is written as [19]

$$
\Lambda(t, 0) \rho(0)=\frac{1}{2} \sum_{i=1}^{2} U_{i}(t) \rho(0) U_{i}^{\dagger}(t),
$$

where

$$
U_{i}(t)=\left(\begin{array}{cc}
\cos (\lambda t) & \mathrm{e}^{-\mathrm{i} \phi_{i}} \sin (\lambda t) \\
-\mathrm{e}^{\mathrm{i} \phi_{i}} \sin (\lambda t) & \cos (\lambda t)
\end{array}\right),
$$

with $i=1,2$ and $\phi_{1}=0, \phi_{2}=\pi . U_{i}(t)=\mathrm{e}^{-\mathrm{i} H_{i} t / \hbar}$ is the time-evolution operator associated with the Hamiltonian $H_{i}=$ $\mathrm{i} \hbar \lambda\left(\sigma_{+} \mathrm{e}^{-\mathrm{i} \phi_{i}}-\sigma_{-} \mathrm{e}^{\mathrm{i} \phi_{i}}\right)$, where $\sigma_{+}, \sigma_{-}$are the qubit raising and lowering operators and $\lambda$ is the qubit-field coupling constant that depends on the field amplitude. The Hamiltonian $H_{i}$ is given in the interaction picture (rotating frame) at the qubit-field resonant frequency $\omega$.

In order to use the non-Markovianity measures introduced above, knowledge of both the dynamical map and the master equation is required. In our model, we directly have the map and we also have to construct the corresponding master equation. To obtain the master equation, starting from the map of equation (1) we follow the procedure proposed in [20] which gives (the details of calculations are reported in the appendix)

$$
\mathrm{d} \rho / \mathrm{d} \tau=L \rho(\tau)=\tan 2 \tau\left(\sigma_{y} \rho \sigma_{y}-\rho\right),
$$

where $\tau=\lambda t$ is a dimensionless time. It is worth noting that this form of master equation, associated with our system, presents a time-dependent rate, $\tan (2 \tau)$, which is the same that has been previously introduced only formally in a general master equation to study non-Markovian behavior [13, 14].

\subsection{The dissipative case}

The model of random external fields described above is non-dissipative and can be generalized to a dissipative case. Although it is not easy to introduce a source of dissipation directly into the map, it is simple to do it into the master equation. We phenomenologically add population damping with rate $\gamma$, in the standard Lindblad form with generator $\gamma \sigma_{-}$[24], into the master equation of equation (3), which now becomes

$$
\begin{aligned}
\mathrm{d} \rho / \mathrm{d} \tau= & L \rho(\tau)=\tan 2 \tau\left(\sigma_{y} \rho \sigma_{y}-\rho\right) \\
& +\tilde{\gamma}\left(\sigma_{-} \rho \sigma_{+}-\rho \sigma_{+} \sigma_{-} / 2-\sigma_{+} \sigma_{-} \rho / 2\right),
\end{aligned}
$$

where $\tilde{\gamma}=\gamma / \lambda$ is a dimensionless decay rate. In the following, we shall use the map of equation (1) and the master equations of equations (3) and (4) to analyze whether the different criteria individuate the same time regions when a non-Markovian behavior occurs.

\section{Comparison among the criteria in the non-dissipative case}

We shall first apply the three non-Markovianity criteria (BLP, $\mathrm{RHP}$ and $\mathrm{ACH}$ ) to the case of non-dissipative random external fields.

\subsection{The BLP criterion}

The BLP criterion is based on the distinguishability of two evolving quantum states quantified by the trace distance [13], that is, $D\left(\rho_{1}(t), \rho_{2}(t)\right)=\frac{1}{2}\left\|\rho_{1}(t)-\rho_{2}(t)\right\|_{1}$, where $\|\hat{A}\|_{1} \equiv$ $\operatorname{Tr} \sqrt{\hat{A}^{\dagger} \hat{A}}, \rho_{i}(t)=\Lambda(t, 0) \rho_{i}(i=1,2)$, whose variation rate is

$$
\sigma(t)=\mathrm{d} D\left(\rho_{1}(t), \rho_{2}(t)\right) / \mathrm{d} t .
$$

The dynamical map $\Lambda(t, 0)$ is non-Markovian, according to BLP, if there exists a pair of initial states $\rho_{1}, \rho_{2}$ such that for some time $t>0$ the distinguishability of the two states increases, that is, $\sigma(t)>0$. This is interpreted as a flow of information from the environment back to the system, which enhances the possibility of distinguishing the two states.

Let us apply this criterion to the model of non-dissipative random external fields. Choosing two arbitrary initial states

$$
\rho_{1}=\left(\begin{array}{cc}
\omega & \alpha \mathrm{e}^{\mathrm{i} \varphi_{1}} \\
\alpha \mathrm{e}^{-\mathrm{i} \varphi_{1}} & 1-\omega
\end{array}\right), \quad \rho_{2}=\left(\begin{array}{cc}
\mu & \beta \mathrm{e}^{\mathrm{i} \varphi_{2}} \\
\beta \mathrm{e}^{-\mathrm{i} \varphi_{2}} & 1-\mu
\end{array}\right)
$$

and substituting them into equation (5), we obtain

$$
\sigma(\tau)=-\sqrt{a} \sin (4 \tau) /|b|,
$$

where $\quad a=(\mu-\omega)^{2}+\left(\alpha \cos \varphi_{1}-\beta \cos \varphi_{2}\right)^{2} \quad$ and $b=\cos ^{2} 2 \tau+\alpha \sin \varphi_{1}-\beta \sin \varphi_{2}$. The sign of this quantity does not depend on the value of the parameters of the initial states and thus permits a general comparison with the other criteria. In particular, it is readily found that $\sigma(\tau)>0$ (i.e. the dynamics exhibits non-Markovianity) when $\pi / 4+k(\pi / 2)<\tau<(k+1) \pi / 2$, where $k$ is a non-negative integer number.

\subsection{The RHP criterion}

The RHP criterion is based on the divisibility of a dynamical map and is independent of the system state. If the map $\Lambda(t, 0)$ is divisible, it satisfies the condition $\Lambda_{(t+\epsilon, 0)}=\Lambda_{(t+\epsilon, t)} \Lambda_{(t, 0)}$ ( $\epsilon$ is a time interval) that is usually attributed to Markovian evolution. It is possible to show that the map $\Lambda(t, 0)$ is completely positive, and then divisible, if and only if $\left(\Lambda_{t+\epsilon, t} \otimes\right.$ $\left.\mathbb{1}_{2}\right)|\Phi\rangle\langle\Phi| \geqslant 0$, where $|\Phi\rangle$ is a maximally entangled state of two qubits (one of them is subject to the map while the other is the isolated ancilla) and $\mathbb{1}_{2}$ is the two-dimensional identity matrix [14]. For a qubit subject to a master equation $\mathrm{d} \rho / \mathrm{d} t=L_{t}(\rho)$, where $L_{t}$ is a Lindblad operator, in the limit of $\epsilon \rightarrow 0$ the solution (dynamical map) of this equation formally tends to $\Lambda_{t+\epsilon, t} \rightarrow \mathrm{e}^{L_{t} \epsilon}$. Expanding this solution up to the first order in $\epsilon$, it is possible to introduce the quantity [14]

$$
g(t)=\lim _{\epsilon \rightarrow 0^{+}} \frac{\|\left[\mathbb{1}_{4}+\epsilon\left(L \otimes \mathbb{1}_{2}\right)\right]|\Phi\rangle\langle\Phi| \|_{1}-1}{\epsilon},
$$

where $\|A\|_{1}$ indicates the trace norm. It is shown that $g(t)>0$ if and only if the original map $\Lambda(t, 0)$ is indivisible, that is, exhibits non-Markovian behavior. 
In our case of non-dissipative random external fields, by identifying $L_{t}$ with that of the master equation of equation (3), we obtain $g(\tau)=-2 \tan 2 \tau$ if $\tan 2 \tau<0$ and $g(\tau)=0$ otherwise. It is immediately seen that a non-Markovian behavior $(g(\tau)>0)$ occurs just in the same temporal regions individuated above by the BLP criterion, that is, $\pi / 4+$ $k(\pi / 2)<\tau<(k+1) \pi / 2$.

\subsection{The ACH criterion}

This criterion is based on the property of complete positivity (divisibility) of the dynamical map deduced through the sign of time-dependent decoherence rates that may appear in the master equation. This criterion is also independent of the system state. Consider a qubit governed by a master equation in the canonical (Lindblad-type) form, in the interaction picture [15]

$$
\begin{aligned}
\frac{\mathrm{d} \rho}{\mathrm{d} \tau}= & \sum_{k} \gamma_{k}(\tau)\left[L_{k}(\tau) \rho L_{k}^{\dagger}(\tau)\right. \\
& \left.-\frac{1}{2} L_{k}^{\dagger}(\tau) L_{k}(\tau) \rho-\frac{1}{2} \rho L_{k}^{\dagger}(\tau) L_{k}(\tau)\right],
\end{aligned}
$$

where the traceless operators $L_{k}(\tau)$, time dependent in general, describe different decoherence channels and $\gamma_{k}(\tau)$ are the corresponding decay rates that can also be time dependent. The different decay channels are orthogonal in the sense that $\operatorname{Tr}\left(L_{j}^{\dagger} L_{k}\right)=\delta_{j k}$. If the $\gamma_{k}(\tau)$ are positive at all times, then the time evolution is completely positive in any time interval with a Markovian behavior. On the other hand, if some of the $\gamma_{k}(t)$ are negative, the time evolution exhibits non-Markovian behavior that can then be naturally characterized by the function $f_{k}(\tau)=\min \left[\gamma_{k}(\tau), 0\right]$ for each decoherence channel [15]. This criterion is conceptually similar to the RHP one and is convenient due to its immediate application once we have the expression of the master equation.

In the master equation of equation (3), associated with our model of a qubit under non-dissipative random external fields, the only (dimensionless) decay rate is $\tan 2 \tau$. Once again we find that the time regions where non-Markovian behavior occurs correspond to the negative values of $\tan 2 \tau$.

The above results show agreement among the three criteria in individuating time regions of non-Markovianity considered here, in the case of non-dissipative random external fields.

\section{Comparison among the criteria in the dissipative case}

We now analyze the RHP and ACH criteria in the case of a qubit subject to random external fields and to population decay, whose master equation is given in equation (4). We do not consider the BLP criterion that requires knowledge of the qubit evolution and therefore the solutions of the master equation of equation (4): this will be treated elsewhere.

The function $g(t)$ of equation (8) of the RHP criterion now becomes

$$
g(\tau)=-\tilde{\gamma} / 2-\tilde{\gamma}_{1}(\tau) / 2+(\sqrt{2} / 4)\left[\tilde{g}_{+}(\tau)+\tilde{g}_{-}(\tau)\right],
$$

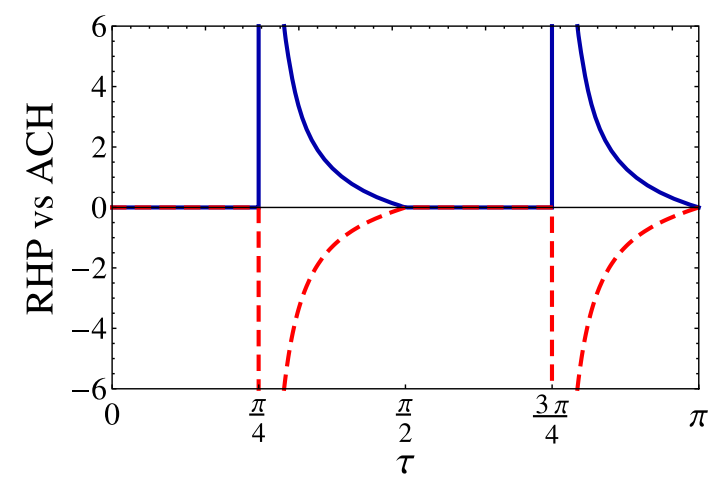

Figure 1. Comparison between the function $g(\tau)$ of the RHP criterion (blue solid line) and the function $f(\tau)$ of the $\mathrm{ACH}$ criterion (red dashed line) as a function of the dimensionless time $\tau$, for a dimensionless decay rate $\tilde{\gamma}=3$. There is non-Markovianity when $g(\tau)>0$ according to RHP and when $f(\tau)<0$ according to ACH.

where

$$
\tilde{g}_{ \pm}(\tau) \equiv\left\{\tilde{\gamma}^{2}+\left[\tilde{\gamma}+\tilde{\gamma}_{1}(\tau)\right]\left[\tilde{\gamma}_{1}(\tau) \pm \sqrt{\tilde{\gamma}^{2}+\tilde{\gamma}_{1}^{2}(\tau)}\right]\right\}^{1 / 2}
$$

and $\tilde{\gamma}_{1}(\tau) \equiv 2 \tan 2 \tau$.

To use the ACH criterion, we put the master equation of equation (4) into the canonical form of equation (9) by using the procedure of [15]; two orthogonal decay channels arise with rates

$$
\tilde{\gamma}_{ \pm}(\tau)=\left(\tilde{\gamma}+2 \tan 2 \tau \pm \sqrt{\tilde{\gamma}^{2}+4 \tan ^{2} 2 \tau}\right) / 2,
$$

and corresponding operators $L_{ \pm}=\sum_{i=1,2} U_{i}^{( \pm)} \sigma_{i} / \sqrt{2}$, where $\sigma_{i}(i=1,2)$ are the usual Pauli matrices and

$$
\begin{aligned}
& U_{1}^{( \pm)}=\frac{i\left(-2 \tan 2 \tau \pm \sqrt{\tilde{\gamma}^{2}+4 \tan ^{2} 2 \tau}\right)}{\sqrt{\tilde{\gamma}^{2}+\left(2 \tan 2 \tau \mp \sqrt{\tilde{\gamma}^{2}+4 \tan ^{2} 2 \tau}\right)^{2}}}, \\
& U_{2}^{( \pm)}=\tilde{\gamma} / \sqrt{\tilde{\gamma}^{2}+\left(2 \tan 2 \tau \mp \sqrt{\tilde{\gamma}^{2}+4 \tan ^{2} 2 \tau}\right)^{2}} .
\end{aligned}
$$

Being $\tilde{\gamma}_{-}(\tau) \leqslant \tilde{\gamma}_{+}(\tau)$ at any time, the non-Markovianity regions according to $\mathrm{ACH}$ are characterized only by the function $f_{-}(\tau)=\min \left[\tilde{\gamma}_{-}(\tau), 0\right]$. From equation (11), the condition $\tilde{\gamma}_{-}(\tau)<0$ is satisfied when $4 \tilde{\gamma} \tan 2 \tau<0$ (i.e. $\pi / 4+k(\pi / 2)<\tau<(k+1) \pi / 2)$. Therefore, the $\mathrm{ACH}$ criterion in the dissipative case individuates non-Markovianity in the same time regions of the previous non-dissipative case.

In this dissipative case, the $\mathrm{ACH}$ criterion evidences non-Markovian behavior in the same time regions individuated by the RHP criterion. This is displayed in figure 1, where it is seen that the function $g(\tau)$ of the RHP criterion is greater than zero exactly when the function $f(\tau)$ of the $\mathrm{ACH}$ criterion is lower than zero.

All the above results are independent of the initial state of the system.

\section{Conclusions}

In this paper, we have analyzed three different criteria (BLP, RHP and ACH) identifying non-Markovian behaviors in a realistic system made of a qubit subject to random external fields, both in a non-dissipative and in a dissipative evolution. We have first exactly obtained the master equation 
corresponding to the qubit dynamical map of random external fields. We point out that the form of the master equation, associated with our system, contains the time-dependent rate $\tan (2 \tau)$ that has been previously inserted only formally into a general master equation to study non-Markovian behavior [13, 14]. We have then phenomenologically introduced population damping directly in the master equation associated with the map of random external fields.

We have found, in the non-dissipative case, that the three criteria agree in individuating non-Markovianity time regions. For the model of random external fields with population decay, both the RHP and ACH criteria individuate the same time regions of non-Markovian behavior.

The results of this paper may provide new insight into the topic of characterizing the non-Markovianity in a realistic open quantum system.

\section{Acknowledgments}

The authors acknowledge Ángel Rivas Vargas for fruitful comments and suggestions.

\section{Appendix. The master equation associated with the model of non-dissipative random external fields}

In this appendix, we summarize the steps to obtain the master equation of equation (3) from the map of random external fields of equation (1) by following the general procedure described in [20].

The general steps are as follows. Let us apply a map $\Lambda(t, 0)$ to the basis operators $G_{i}=\sigma_{i} / \sqrt{2}(i=0, \ldots, 3)$, where $\sigma_{0}=\mathbb{1}$ and the remaining $\sigma_{i}$ are the Pauli matrices, and define a matrix $F$ with elements $F_{k l} \equiv \operatorname{Tr}\left[G_{k} \Lambda(t, 0)\left(G_{l}\right)\right]$. The idea is to construct a matrix $\dot{F} F^{-1}$ (or, more generally, $\dot{F} \tilde{F}$ if $F$ is not invertible). In our case $F$ is invertible and it is possible to calculate the matrix $R$, with elements defined by

$$
R_{a b}=\sum_{r s}\left(\dot{F} F^{-1}\right)_{r s} \operatorname{tr}\left[G_{r} \tau_{a}^{\dagger} G_{s} \tau_{b}\right]
$$

where $\tau_{a}=\left|\alpha_{1}\right\rangle\left\langle\alpha_{2}\left|, \tau_{b}=\right| \beta_{1}\right\rangle\left\langle\beta_{2}\right|$, with $\left|\alpha_{1}\right\rangle,\left|\alpha_{2}\right\rangle$ and $\left|\beta_{1}\right\rangle$, $\left|\beta_{2}\right\rangle$ being the qubit basis states $|1\rangle,|2\rangle$. The general expression of the master equation is then

$$
L(\rho(\tau))=\dot{\rho}(\tau) \equiv \sum_{a b} R_{a b}(t) \tau_{a} \rho(t) \tau_{b}^{\dagger}
$$

where the operators $\tau$ are $\tau_{0}=|2\rangle\left\langle 2\left|=\sigma_{+} \sigma_{-}, \tau_{1}=\right| 1\right\rangle\langle 1|=$ $\sigma_{-} \sigma_{+}, \quad \tau_{2}=|2\rangle\langle 1|=\sigma_{+}$and $\tau_{3}=|1\rangle\langle 2|=\sigma_{-}$, with $\sigma_{ \pm}=$ $\left(\sigma_{1} \pm \mathrm{i} \sigma_{2}\right) / 2$. In our case of random external fields with the map given in equation (1), we obtain the matrix $F$

$$
F=\left(\begin{array}{cccc}
1 & 0 & 0 & 0 \\
0 & \cos 2 \tau & 0 & 0 \\
0 & 0 & 1 & 0 \\
0 & 0 & 0 & \cos 2 \tau
\end{array}\right)
$$

from which one easily obtains the matrices $F^{-1}, \dot{F}$ and therefore the matrix $\dot{F} F^{-1}$. Choosing the basis $\{|2\rangle\langle 2|| 1\rangle,\langle 1|| 2\rangle,\langle 1|| 1\rangle,\langle 2|\}$ and using equation (A.1), we find the $R$ matrix as

$$
R=\left(\begin{array}{cccc}
-\tan 2 \tau & -\tan 2 \tau & 0 & 0 \\
-\tan 2 \tau & -\tan 2 \tau & 0 & 0 \\
0 & 0 & \tan 2 \tau & -\tan 2 \tau \\
0 & 0 & -\tan 2 \tau & \tan 2 \tau
\end{array}\right)
$$

Finally, using equation (A.2) we obtain the desired master equation

$$
\mathrm{d} \rho / \mathrm{d} \tau=\tan 2 \tau\left(\sigma_{y} \rho \sigma_{y}-\rho\right) .
$$

\section{References}

[1] Rivas A and Huelga S F 2011 Open Quantum Systems. An Introduction (Heidelberg: Springer)

[2] Chruscinski D and Kossakowski A 2010 Phys. Rev. Lett. 104070406

[3] Chruscinski D, Kossakowski A and Rivas A 2011 Phys. Rev. A 83052128

[4] Bellomo B, Lo Franco R and Compagno G 2007 Phys. Rev. Lett. 99160502

[5] Lo Franco R, D’Arrigo A, Falci G, Compagno G and Paladino E 2012 Phys. Scr. T147 014019

[6] Lo Franco R, Bellomo B, Maniscalco S and Compagno G 2012 arXiv: 1205.6419

[7] Bellomo B, Lo Franco R, Maniscalco S and Compagno G 2008 Phys. Rev. A 78060302

[8] Bellomo B, Lo Franco R, Maniscalco S and Compagno G 2010 Phys. Scr. T140 014014

[9] Yu T and Eberly J H 2009 Science 323598

[10] Lai C W, Maletinsky P, Badolato A and Imamoglu A 2006 Phys. Rev. Lett. 96167403

[11] Plenio M B and Knight P L 1998 Rev. Mod. Phys. 70101

[12] Aharonov D, Kitaev A and Preskill J 2006 Phys. Rev. Lett. 96050504

[13] Breuer H P, Laine E M and Piilo J 2009 Phys. Rev. Lett. 103210401

[14] Rivas A, Huelga S and Plenio M B 2010 Phys. Rev. Lett. 105050403

[15] Andersson E, Cresser J D and Hall M J 2010 arXiv:1009.0845 [quant-ph]

[16] Liu B H, Li L, Huang Y F, Li C F, Guo G C, Laine E M, Breuer H P and Piilo J 2011 Nature Phys. 7931

[17] Zeng H, Tang N, Zheng Y and Wang G 2011 Phys. Rev. A 84032118

[18] Haikka P, Cresser J D and Maniscalco S 2011 Phys. Rev. A 83012112

[19] Lo Franco R, Andersson E, Bellomo B and Compagno G 2012 Phys. Rev. A 85032318

[20] Andersson E, Cresser J D and Hall M J 2007 J. Mod. Opt. 541695

[21] Bellomo B, Lo Franco R, Andersson E, Cresser J D and Compagno G 2012 Phys. Scr. T147 014004

[22] Alicki R and Lendi K 2007 Quantum Dynamical Semigroups and Applications (Berlin: Springer)

[23] Zyczowski K, Horodecki P, Horodecki M and Horodecki R 2001 Phys. Rev. A 65012101

[24] Nielsen Chuang 2000 Quantum Computation and Quantum Information (Cambridge University Press: Cambridge) 